\title{
CROSS SENTENCE ALIGNMENT BASED ON SINGULAR VALUE DECOMPOSITION
}

\author{
ANNA HO $^{1}$, FAI WONG ${ }^{1}$, FRANCISCO DE OLIVEIRA ${ }^{1}$, YIPING LI ${ }^{1}$ \\ ${ }^{1}$ Faculty of Science and Technology of University of Macau, PO Box 3001 Macao SAR \\ E-MAIL: ma36560@umac.mo, derekfw@umac.mo, olifran@umac.mo, ypli@umac.mo
}

\begin{abstract}
:
This paper describes a way of performing alignment of Portuguese-Chinese bilingual pairs from the given two documents. Extracting the alignment pairs is a critical step for building Portuguese-Chinese bilingual corpus for Example Based Machine Translation Systems (EBMT). In short, the proposed alignment system performs four steps: break down the document into sentences level, score each pair of sentence by different features, apply Singular Value Decomposition on the results, extract the aligned pair base on a similarity function.
\end{abstract}

\section{Keywords:}

Bilingual document alignment; singular value decomposition; segmentation; cross sentence alignment

\section{Introduction}

Recently, most of the machine language translation related research only focuses on the algorithm of English-Chinese or structurally similar languages. Relatively, Portuguese-Chinese or structurally dissimilar languages has less related resources. Considering there is still improvements can be made in Portuguese-Chinese machine translation algorithm, this paper focus on describing new issues on this related field. Since alignment of bilingual corpus is a significant process in developing an Example Based Machine Translation System, therefore the following sessions in this paper expose the corresponding alignment process step by step. The corpus chosen for testing this algorithm comes from different categories, such as legislative laws, magazines and government official gazette. As the corpus comes from different areas, the proposed alignment algorithm employs the Singular Value Decomposition (SVD) technique in the algorithm so that it is capable to handle not only one-to-one parallel mapping cases, but also cross level sentence alignment cases as shown in Figure 1.

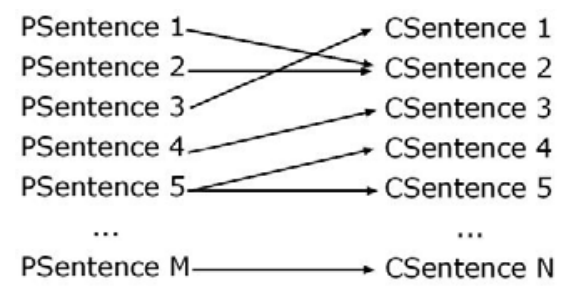

Figure 1. Possible Cross Level Sentence Alignment

\section{Review}

Currently, existing literature already provides many different approaches on aligning bilingual documents. Those techniques can be generally classified into three types: lexicon based [1-2], statistical based [3-4], and combination of lexicon and statistical based [5]. Lexicon based techniques mainly makes use of the dictionary to perform the alignment procedure. On the other hand, statistical based approach usually relies on the statistical issues such as length ratio of the sentences in bilingual languages. The combination approach mixes the above two methods in order to gain their advantages thus maximize accuracy of the resulting output.

However, the techniques mentioned in the above paragraph are solutions mainly focus on the alignment of structurally similar languages, for example, the research works of Melamed [6] and Fung, et al. [7]. While applying those techniques directly into Portuguese and Chinese documents cannot effectively align comparable or unparallel bilingual corpus with dissimilar language structure. One of our difficulties is to solve the problem of dissimilarity of sentence structure, which cause our approach cannot rely on the appearance of the bilingual sentence. Another difficulty is that meanings expressed in different Chinese words may actually refer to the same Portuguese word. For example, the word “correio”_(post) can find a translation “郵包” in the dictionary, but in the corresponding bilingual sentence, it consists the word “郵 
件”, though the expression in Chinese is a little bit different, but the two words should be the same. The approaches of the above mentioned techniques assume the corpus is parallel. Since without assuming the corpus is parallel, we leave this problem for the algorithm to handle, so the process should have intelligence to align its correct translated sentence.

The main difference to the mentioned algorithms is that we employ the Singular Value Decomposition (SVD) techniques in this proposed approach. This techniques has widely used in document summarization [8] and genes related research work. Recently, Oliveira, et, al [9] shows that applying SVD is also successful in handling word sense similarity measuring. This is because SVD can reduce the dimensionality of the term-document matrix, removes random noise from the matrix and it is sensitive to high-order co-occurrence information which is ignored by the cosine similarity measures, and therefore SVD assists the algorithm to have a better measurement in similarity. In this proposed approach, we try to make use of these characteristics of SVD to enhance the accuracy of cross sentence alignment. The following sections are organized as: alignment approach, experiments and conclusion.

\section{Alignment approach}

The proposed alignment method mainly consists of five steps: corpus pre-processing, data construction, scoring features, apply singular value decomposition and align the paired bilingual sentences. The flow of the approach is shown in Figure 2.

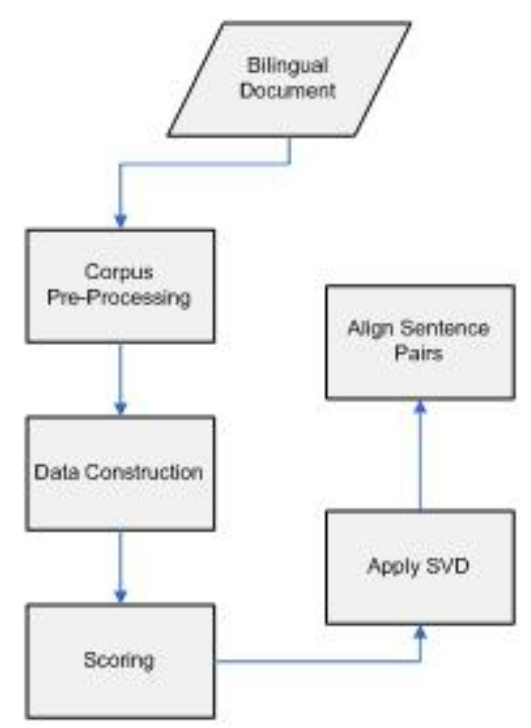

Figure 2. Procedure of alignment

\subsection{Corpus pre-processing}

This is the first step of our approach It takes Chinese and Portuguese documents as inputs, according to the incoming language, the document will be processed by the corresponding sentence segmentation module. The segmentation module is responsible to split the document into sentence vectors according to the punctuation (full stop, exclamation mark and question mark).

After splitting up into sentence vectors, the process filters out non-informative words in the Portuguese sentences according to the given stop word database. Those stop words include Portuguese word such as "que”, "para”, "de", etc. Those words cannot find out corresponding Chinese translation, which will cause accuracy problems in retrieving the alignment results. In order to perform the filtering action, the sentences must be represented in its original format, therefore a morphologically analyzing module is employed. Here is an example result of this process: "Eu trabalho em Universidade de Macau" (I work in University of Macau) becomes " $E u$ trabalhar Universidade Macau”.

\subsection{Statistical relationship matrix construction}

After the preparation of the bilingual sentence vectors, this step constructs the statistical relationship matrix of this algorithm. In order to apply SVD to the statistical data, this step presents the statistics in a Statistical Relationship Matrix (SRM), which represents the relationship of the sentence pairs, as shown in Figure 3. Each entry in the matrix shows how each Portuguese sentence is related to each Chinese sentence.

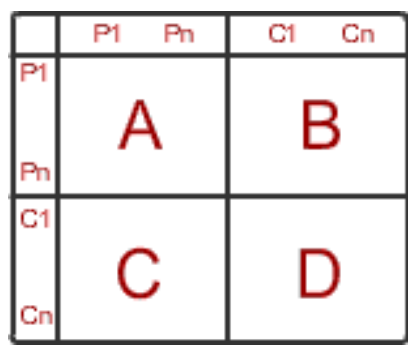

Figure 3. Statistical Relationship Matrix

In Figure 3, entries in Zone A and Zone D actually are the relationship entries for Portuguese-Portuguese sentences and Chinese-Chinese sentences respectively, these zones only gives a score one to the identical sentence pair such as entry in [P1, P1] or [C1, C1]. For the other entries in these two zones, such as [P1, P2] or [C1, C2], the process gives 
zero. For Zone $\mathrm{B}$ and Zone $\mathrm{C}$, these are the entries calculated by formula (1). This formula includes four types of features: lexicon matching, punctuation matching, length ratio measuring and digit groups matching. Each of the features has assigned their own weights that are $l_{1}, l_{2}, l_{3}$ and $l_{4}$ respectively; each sub-formula, $S_{1}, S_{2}, S_{3}, S_{4}$ and $S_{5}$ describes in the following sessions respectively.

$$
\text { score }[i, j]=l_{1} S_{1} S_{4}+l_{2} S_{2}+l_{3} S_{3}+l_{4} S_{5} \leq 1
$$

\subsection{Lexicon matching}

Lexicon matching is defined as by giving a Portuguese-Chinese dictionary and two sentences, Sentence $_{p}$ as Portuguese sentence and Sentence ${ }_{c}$ as Chinese sentence, this feature can figure out how well that Sentence $e_{p}$ can be translated into Sentence ${ }_{c}$. It is defined as formula (2), (3), (4) and (5).

$$
\begin{aligned}
& S_{1}=\frac{\text { fullMatch }_{p, c}+\text { partialMatch }_{p, c}}{\# \text { foundMeanings }} \leq 1 \\
& \text { FullMatch }_{p, c}=\sum_{k}{\text { foundFullMatch }\left(\text { WPort }_{k}\right)}_{\text {PartialMatch }_{p, c}=\sum_{k} \operatorname{Max}\left(f_{P M}\right)} \\
& f_{P M}=\frac{\sum_{i=1}^{4} \frac{\text { matched }_{\text {total }}^{4}}{\sum_{i=1}^{4} n w_{i}}, w_{i}}{\text { to }_{i}=\text { gram }_{-} \text {weight }}
\end{aligned}
$$

For each entry $[p, c]$, as shown in formula (2), the process calculates the lexicon matching score by considering two cases: FullMatch and PartialMatch.

FullMatch is the case in which a Portuguese word in Sentence $_{p}$ can find a corresponding meaning from the dictionary. This meaning considered as a set of Chinese characters and in a specific order which exists in Sentence ${ }_{c}$. For example:

empregado ter direito de acesso a o seguinte; / 僱員的查閱 權包括;

(Worker has right to access the following;)

the word “empregado” (worker) means “僱員” totally included in the Chinese sentence, then it is said that "empregado" is a FullMatch case. As in formula (3) shows, for each Portuguese word WPort ${ }_{k}$ in Sentence , $_{\text {, if }}$ it can find an exact meaning from Sentence ${ }_{c}$, the foundFullMatch function gives one else zero. After parsing all Portuguese words, the formula will find out how many WPort gains an exact match.

For those Portuguese words that do not fulfill the FullMatch case, they fall into the PartialMatch case. For example,

Direito de posse de a conta de correio electronico/電郵帳戶 之擁有權

(Ownership of email account)

from the provided dictionary, the word "correio" (post office) has many different meanings such as “郵包” and “郵彪員”. The process calculates the partial matching score for all the found meanings and selects the highest score as shown in formula (4). As shown in formula (5), this calculates the probability of how this meaning exits in the target Chinese sentence. As shown in Eck., et al. [10], $\mathrm{N}$-gram has a characteristic of preserving sequential order. By modify this concept to Chinese character N-gram based approach in this case can preserve the sequential order of the characters in the Chinese meaning. However, a sub-phrase mismatch case may be happened, and the case is defined as follow:

P1: Vamos encontrar-nos a porta do teatro/ C1: 我們在劇 院門口

(We are at the cinema entrance)

P2: Vamos encontrar-nos a porta do teatro ou vou buscar-te a casa/ C2: 我們在劇院門口碰面還是我去你家接你

(Whether we meet at the cinema entrance or I go to join you at your house)

Ideally, P1 should be aligned to $\mathrm{C} 1$ and $\mathrm{P} 2$ should be aligned to C2. However, P1 is actually a sub-phrase of P2 and so as C1 and C2. In this case, P1 may accidentally align with C2, because C2 can satisfy all the meaning found from the Portuguese words of P1. To avoid this mistake, formula (6) is introduced. The idea is to punish the sub-phrased Portuguese sentence when processing with the longer Chinese sentences. In the above example, when P1 is trying to match with $\mathrm{C} 2$, since $\mathrm{C} 2$ is longer, there are more remaining Chinese characters, so that this can make the final lexicon matching score of [P1, C2] lower.

$$
S_{4}=1-\frac{\text { non_matched_chinese_char }}{\text { total_chinese_char }}
$$




\subsection{Punctuation matching}

This feature is responsible to show how Sentence $e_{p}$ and Sentence $_{c}$ can be matched in the sense of punctuations. In the research done by Chuang, et al. [11] shows that punctuations are sometimes an important key of showing relationships when lexicon knowledge is not enough. In this proposed alignment approach, punctuation matching acts as an assistant feature, so the punctuation matching formula used in this approach is shown as formula (7). The idea is to count how many punctuations in Sentence ${ }_{p}$ exist also in Sentence . $^{2}$

$$
S_{2}=\sum_{j} \text { PuncMatch }\left(\text { punc }_{j}, \text { sentence }_{c}\right)
$$

\subsection{Length ratio measuring}

This feature falls on the statistical approach. Since statistical approach requires very little linguistic knowledge which can assist the algorithm to gain extra knowledge that is failed in lexicon matching feature. As in the previous paragraphs has told, Portuguese and Chinese are in different linguistic structure, thus the length ratio of them is not 1:1. According to the research experiment done by Li et al. [12], Portuguese-Chinese alignment systems can have a good result when mean $=0.694$. Therefore, the mathematical definition of this feature becomes formula (8), (9) and (10).

$r=\mid \frac{\text { length }}{\text { length }_{p}}-$ mean $\mid$

level $=\left\{\begin{array}{l}1, r \geq 1 \\ 1-r\end{array}\right.$

$S_{3}=1-$ level

For every pair of Portuguese and Chinese sentence, the process calculates the corresponding length ratio as defined in formula (8). As we treat the mean as a referenced norm. Therefore when $r$ is greater than 1 , then $S_{3}$ is 0 . On the other hand, if the ratio of the sentence pair tends to the mean, it stats that they have a greater chance to be aligned, so in this case, $S_{3}$ should tends to 1 .

\subsection{Digit groups matching}

This is a special treatment for those texts with many numeric descriptions and decimal numbers. In this situation, numeric descriptions become an important key to align the sentences. This feature score is calculated by formula (11). The following example can clarify this simple idea.

$$
S_{5}=\frac{\text { matchGroup }}{\text { totalDigitGroups }}
$$

P1: Por seu turno , a taxa de cobertura de a exportacao sobre a importacao descer de $80.9 \%$, em 2004 , para 63.3 $\%$ em 2005./C1: 進出口比率則由 2004 年的 $80.9 \%$ 下跌至 2005 年的 $63.3 \%$.

(The ratio of import and export rate decreases from 80.9\% in 2004 to $63.3 \%$ in 2005.)

P2: Por seu turno , a taxa de cobertura de a exportacao sobre a importacao descer de $80 \%$, em 2006 , para $36.3 \%$ em 2007./C2: 進出口比率由 2006 年的 $80 \%$ 下跌至 2007 年的 $36.3 \%$.

(The ratio of import and export rate decreases from $80 \%$ in 2006 to $36.3 \%$ in 2007.)

The above sentences, there are totally 4 digit groups in P1; they are 80.9, 2004, 63.3, 2005. A digit group treats the order of the numbers and the position of decimal points strictly. Without this feature, P1 may align wrongly to C2, since lexicon and length ratio feature may get similar score. This feature will gives 0 to $S_{5}$ when processing $\mathrm{P} 1$ and C2, thus assists the algorithm to align P1 to C1 correctly.

\subsection{Application of Singular Value Decomposition}

After processing all the possible pairs of Portuguese and Chinese sentences, all the entries in the SRM have been calculated. In this stage, SVD technique is applied to the SRM, the procedure can be briefly shown in Figure 4 . In the figure, $W$ represents the original SRM. The key in this procedure is choosing the rank that is the $R$ in the figure. After a lot of testing in the system, $\operatorname{ran} k=\frac{K}{2}$ gives the best result in most cases. Therefore, finally we have the dimensionally reduced SRM, that is $W^{\prime}=U^{\prime} S^{\prime} V^{T}$, where $U^{\prime}, S^{\prime}$ and $V^{T}$, has $\operatorname{rank}=\frac{K}{2}$. 


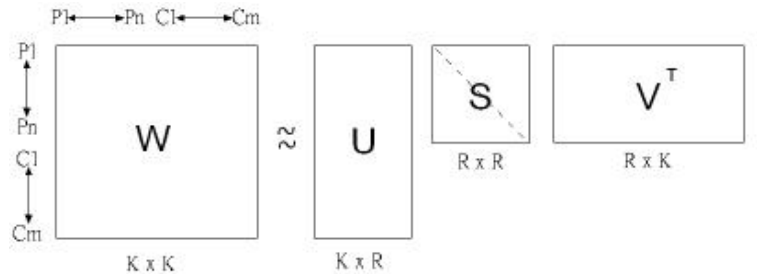

Figure 4. Procedure of applying SVD

Consider an example: assuming there are three pair of sentences, P1 align with C1, P2 align with C2 and P3 align with C3. The part of the original SRM is shown in Table 1. Entry in [P1, C1] and [P1, C2] is the same, and [P2, C1] is slightly greater than [P2, C2]. With this matrix, apply cosine similarity measurement will cause in accurate alignment for there contains noisy and confused information in the matrix. After the application of SVD, the part of the reduced SRM is shwonin Table 2, which contains the most accurate expression showing the relationship of each pair of the bilingual sentence.

Table 1. Original SRM before SVD

\begin{tabular}{|l|l|l|l|}
\hline & C1 & C2 & C3 \\
\hline P1 & 0.35 & 0.35 & 0.13 \\
\hline P2 & 0.30 & 0.295 & 0.18 \\
\hline P3 & 0.1 & 0.15 & 0.27 \\
\hline
\end{tabular}

Table 2. Reduced SRM after SVD

\begin{tabular}{|l|l|l|l|}
\hline & C1 & C2 & C3 \\
\hline P1 & 0.34 & 0.70 & 0.1 \\
\hline P2 & 0.23 & 0.48 & 0.13 \\
\hline P3 & 0.09 & 0.11 & 0.79 \\
\hline
\end{tabular}

The above matrix example is just only part of the matrix, but it already shows that without SVD, the sub-matrix made cause confusion to the algorithm when calculating the cosine similarity.

\subsection{Sentence pairing}

Finally comes to the last step of the alignment system. With the new SRM (SRM') the system applies a cosine similarity measurement on it and forms an alignment matrix (AM). The application of the similarity measurement is shown as figure 5 .

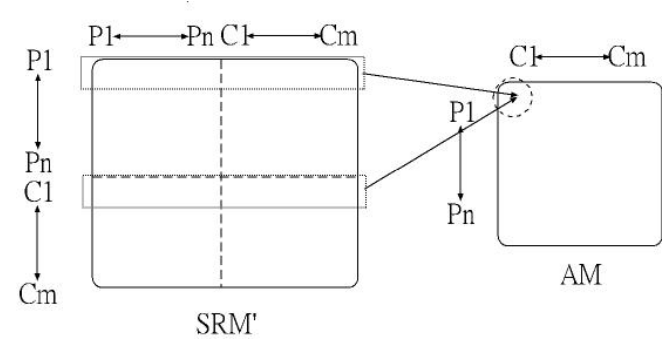

Figure 5. Cosine similarity measurement on SRM'

For each Portuguese rows $P_{i}$ and Chinese rows $C_{j}$, the similarity score of this pair will insert into the AM in the corresponding entry which is $[i, j]$. After every pair of sentences has been calculated their own similarity score, according to the AM, the system now do the alignment. The aligning rules is simple, for each row in the AM, the system choose the highest value, for example [1, 2]. Then the system will check if column 2 also has the highest value at row 1 . If true, then [1, 2] is an accepted alignment, otherwise, row 1 has to choose the second highest value, and perform the column again, until the column check is succeed. This is for the overall case, to ensure the result to be more reliable and accurate, the system filter out the similarity degree which less than a certain threshold, so that the remaining alignment pairs are said to be confirmed alignment.

\section{Experiments}

In this paper, the experiments are done by using the following corpus: Macao Year Book 2006 (corpus 1), parts of Educational Digest Volume 14 and 17 (corpus 2), administrative laws of Macao SAR (corpus 3), Macao SAR Policy Address 2007 (corpus 4) and internal staff regulations of a company (corpus 5). All of the sources are provided with Portuguese and Chinese. Table 3 shows the number of sentences of the bilingual documents.

Table 3. Size of used corpus

\begin{tabular}{|c|c|c|}
\hline & Portuguese & Chinese \\
\hline Corpus 1 & 89 & 72 \\
\hline Corpus 2 & 140 & 108 \\
\hline Corpus 3 & 484 & 484 \\
\hline Corpus 4 & 145 & 107 \\
\hline Corpus 5 & 146 & 132 \\
\hline
\end{tabular}

The experiment is done under the following feature weights: 0.6 for lexicon matching, 0.1 for punctuation, 0.2 for length ratio and 0.1 for digit group matching. The results are shown in Table 4 . Threshold is a value that 
guarantees the system can capture $70 \%$ sentences of the corresponding corpus. Sentence pair with a similarity degree higher or equal to the threshold will be captured and is confirmed to be aligned pairs.

Table 4. Experiment results of different corpus

\begin{tabular}{|l|c|c|c|c|}
\hline & Threshold & $\begin{array}{c}\text { Sentences } \\
\text { captured }\end{array}$ & Incorrect & $\begin{array}{c}\text { Accuracy } \\
(\%)\end{array}$ \\
\hline Corpus 1 & 0.197808 & 63 & 2 & 96.83 \\
\hline Corpus 2 & 0.115949 & 98 & 10 & 89.80 \\
\hline Corpus 3 & 0.066476 & 339 & 48 & 85.84 \\
\hline Corpus 4 & 0.100871 & 102 & 16 & 84.31 \\
\hline Corpus 5 & 0.138195 & 104 & 4 & 96.15 \\
\hline
\end{tabular}

\section{Conclusion}

Applying SVD to enhance the accuracy of cross level alignment is primarily to be proved that it is successful in this paper. For sure, there is still room to improve that results and algorithm, such as include named entity recognition, add learning module so that this can keep less dependence on lexicon resources.

\section{Acknowledgements}

This research work is supported by the University of Macau and INESC-Macau.

\section{References}

[1] Kay. M., and Roscheisen. M., "Text-Translation Alignment”, Computational Linguistics, pp. 121-142, 1993.

[2] Chen. S. F., "Aligning Sentences in Bilingual Corpora Using Lexical Information”, Proceedings of $31^{\text {st }}$ Annual Meeting of the Association for Computational Linguistics. Columbus, pp. 9 - 16, 1993.

[3] Brown. P. F., Lai. J. C. and Mercer. R. L., "Aligning Sentences in Parallel Corpora”, 29th Annual Meeting of the Association for Computational Linguistics, Berkeley, CA, USA, pp. 169-176, 1991.

[4] Gale. W. A. and Church. K. W., "A program for aligning sentences in bilingual corpora”, Computational Linguistics, Vol. 19, pp. 75-102, 1993.

[5] Oliveira. F., Wong. F., Tang C. W., and Li. Y. P., "Extraction of aligned sentence pairs from Portuguese and Chinese documents", Proceedings of Symposium on Applied Science and Technology in Macau, Macau, pp. 28-34, 2004.

[6] Melamed. I. D., "Bitext Maps and Alignment Via Pattern Recognition”, Computational Linguistics, pp.
$107-130,1999$.

[7] Fung. P. and Church. K. W., "Kvec: A New Approach for Aligning Parallel Texts”, Proceedings of COLING 94, Kyoto, Japan, pp. 1064 - 1102, 1999.

[8] Qin. B., Liu. T., and Zhang. Y., "Research on multi-document summarization based on latent semantic indexing”, Journal - Harbin Institute of Technology - English Edition, China, Vol 12, Part 1, pp. 91-94, 2005.

[9] Oliveira. F., Wong. F., Ho. A., Li. Y. P., and Dong. M. C., "Overcoming data sparseness problem in Statistical Corpus based Sense Disambiguation”, Proceedings of the Tenth International Conference on Enhancement and Promotion of Computational Methods in Engineering and Science, Sanya, China, pp. 1099-1104, 2006

[10] Eck. M., Vogel. S., and Waibel. A., "Low Cost portability for Statistical Machine Translation based on N-gram Frequency and TF-IDF”, International Workshop on Spoken Language Translation 2005, Pittsburgh, PA, USA, 2005

[11] Chuang. T. C. and Yeh. K. C., “Aligning Parallel Bilingual Corpora Statistically with Punctuation Criteria”, Computational Linguistics and Chinese Language Processing, Vol 10, No. 1, pp. 95-122, 2005.

[12] Li. Y. P., Pun. C. M. and Wu. F., "Portuguese-Chinese Machine Translation in Macao", Proceedings of Machine Translation, SUMMIT VIT'99, Singapore, pp. 236-243, 1999. 